\section{POS0390 SIMULTANEOUS ASSESSMENT OF JOINT AND VASCULAR INFLAMMATION BY PET-CT IN TOFACITINIB-TREATED PATIENTS WITH RHEUMATOID ARTHRITIS: A PROSPECTIVE STUDY}

A. Hamar $^{1}$, Z. Hascsi ${ }^{2}$, A. Pusztai ${ }^{1}$, M. Czókolyová ${ }^{1}$, E. Végh ${ }^{1}$, Z. Pethö ${ }^{1}$ K. Gulyás ${ }^{1}$, B. Soós ${ }^{1}$, G. Kerekes ${ }^{3}$, É. Szekanecz ${ }^{4}$, K. Hodosi', S. Szántó', G. Szücs ${ }^{1}$, T. Seres ${ }^{5}$, Z. Szekanecz ${ }^{1}$, S. Szamosi ${ }^{1}$. ${ }^{1}$ University of Debrecen, Rheumatology, Debrecen, Hungary; ${ }^{2}$ Scanomed Ltd., PET/CT, Debrecen, Hungary; ${ }^{3}$ University of Debrecen, Medicine, Debrecen, Hungary; ${ }^{4}$ University of Debrecen, Oncology, Debrecen, Hungary; ${ }^{5}$ University of Colorado Anschutz Medical Campus, Anesthesiology, Aurora, Co, United States of America

Background: Rheumatoid arthritis (RA) has bene associated with atherosclerosis and cardiovascular (CV) disease. 18F-fluorodeoxyglucose positron emission tomography/computed tomography $\left({ }^{18} \mathrm{FDG}-\mathrm{PET} / \mathrm{CT}\right)$ is suitable to detect synovial and vascular inflammation. Tofacitinib has been used to effectively treat RA. Objectives: We wished to assess the effects of tofacitinib treatment on synovitis and vascular inflammation simultaneously by ${ }^{18} \mathrm{FDG}-\mathrm{PET} / \mathrm{CT}$.

Methods: Thirty RA patients with active disease were treated with either $5 \mathrm{mg}$ bid or $10 \mathrm{mg}$ bid tofacitinib and evaluated at baseline and after 6 and 12 months. We determined DAS28, CRP, IgM rheumatoid factor (RF) and anti-cyclic citrullinated peptide (aCCP) levels. All patients underwent 18F-fluorodeoxyglucose positron emission tomography/computed tomography (FDG-PET/CT) in order to determine vascular and synovial inflammation in five aortic segments and five articular regions, respectively. In the joints, mean (SUV ${ }_{\text {nend }}$ ) and maximum standard uptake values (SUV ${ }_{\text {ox }}$ ), while in the aorta, mean (TBR ${ }_{\text {max }}$ ) and maximum target-to-background ratios $\left(\mathrm{TBR}_{\max }\right)$ were determined. Carotid intima-media thickness (IMT), arterial stiffness (PWV) and endothelial dysfunction (FMD) were determined by ultrasound.

Results: One-year tofacitinib treatment significantly attenuated vascular and synovial inflammation as visualized by PET/CT. Articular SUV ${ }_{\text {mean }}(p=0.010)$, SUV $_{\text {max }}(p=0.001)$, as well as aorta TBR Tax $_{\text {max }}(p<0.001)$ significantly decreased over time. Synovial inflammation as determined by PET/CT variably and positively associated with aCCP, RF, CRP, ApoB, lipoprotein A (LpA), IMT and PWV. Vascular inflammation (TBR ${ }_{\text {max }}$ ) inversely correlated with $\mathrm{HAQ}$ and positively with ESR, ApoA, and PWV. Uni- and multivariable analyses suggested that articular SUV values were independently associated with CRP, ApoB, LpA, IMT and PWV,

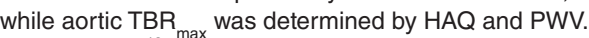

Conclusion: ${ }^{18} \mathrm{~F}-\mathrm{PET} / \mathrm{CT}$ is suitable to simultaneously assess synovial and vascular inflammation in RA. One-year tofacitinib treatment dampened inflammation. $\mathrm{PET} / \mathrm{CT}$ changes were associated with markers of systemic inflammation, atherogenic lipids, carotid atherosclerosis and arterial stiffness.

REFERENCES

[1] Gotthardt M, Bleeker-Rovers CP, Boerman OC, Oyen WJ. Imaging of inflammation by $\mathrm{PET}$, conventional scintigraphy, and other imaging techniques. $J$ Nucl Med. 2010;51(12):1937-49.

[2] Bucerius J, Hyafil F, Verberne HJ, Slart RH, Lindner O, Sciagra R, et al. Position paper of the Cardiovascular Committee of the European Association of Nuclear Medicine (EANM) on PET imaging of atherosclerosis. Eur J Nucl Med Mol Imaging. 2016;43(4):780-92.

Acknowledgements: This research was supported by the European Union and the State of Hungary and co-financed by the European Social Fund in the framework of TAMOP-4.2.4.A/2-11/1-2012-0001 'National Excellence Program' (Z.S.); by the European Union grant GINOP-2.3.2-15-2016-00015 (G.P., G.T. and Z.S.) and by the Pfizer Investigator Initiated Research Grant no. WI188341 (Z.S.). Disclosure of Interests: Attila Hamar: None declared, Zsolt Hascsi: None declared, Anita Pusztai: None declared, Monika Czókolyová: None declared, Edit Végh: None declared, Zsófia Pethö: None declared, Katalin Gulyás: None declared, Boglárka Soós: None declared, György Kerekes: None declared, Éva Szekanecz: None declared, Katalin Hodosi: None declared, Sándor Szántó Speakers bureau: Abbvie, MSD, Novartis, Consultant of: Abbvie, MSD, Novartis, Gabriella Szücs Speakers bureau: Boehringer, Actelion, Roche, Consultant of: Boehringer, Actelion, Roche, Tamas Seres: None declared, Zoltán Szekanecz Speakers bureau: Abbvie, Pfizer, Roche, Novartis, Lilly, Sager, Janssen, Consultant of: Pfizer, Abbvie, Roche, Novartis, Grant/research support from: Pfizer, Szilvia Szamosi Speakers bureau: Roche

DOI: 10.1136/annrheumdis-2021-eular.2473

\section{POS0391 ASSESSING SENESCENT HUMAN LYMPH NODE STROMAL CELLS DURING RHEUMATOID ARTHRITIS DEVELOPMENT}

T. de Jong ${ }^{1,2}$, J. W. Bolt ${ }^{1,2}$, J. F. Semmelink ${ }^{1,2}$, L. van Baarsen ${ }^{1,2} .{ }^{1}$ Amsterdam UMC, University of Amsterdam, Department of Rheumatology \& Clinical Immunology and Department of Experimental Immunology, Amsterdam, Netherlands; ${ }^{2}$ Amsterdam UMC, Amsterdam Rheumatology \& Immunology Center (ARC), Amsterdam, Netherlands
Background: Cellular senescence is a state of proliferation arrest of cells. The persistence and accumulation of senescent cells has been implicated in the pathogenesis of age-related diseases like rheumatoid arthritis (RA). Clinical disease is preceded by loss of immune tolerance and autoimmunity. Lymph node stromal cells (LNSC) are important regulators of this tolerance. Therefore, senescent LNSC may affect tolerance and the development of systemic autoimmunity. Objectives: To determine the extent of cellular senescence of LNSC during early phases of systemic autoimmunity.

Methods: We included individuals with arthralgia without any evidence of arthritis who were positive for IgM rheumatoid factor (IgM-RF) and/or anti-citrullinated protein antibodies (ACPA; RA-risk group), early arthritis patients (ACR/EULAR 2010 criteria; disease duration $<1$ year) and seronegative healthy controls. All study subjects underwent ultrasound-guided inguinal lymph node biopsy. LNSC were isolated and cultured from freshly collected lymph node needle biopsies and passages 0-9 were used for experiments. Cellular senescence was assessed by measuring cell size, granularity, autofluorescence, senescence-associated gene expression levels, DNA damage and senescence-associated $\beta$-galactosidase (SA $\beta$-gal) activity.

Results: Preliminary flow cytometry data shows that the cell size and autofluorescence of LNSC from RA patients $(n=11)$ and RA-risk individuals $(n=7)$ is increased compared with healthy LNSC $(n=7)$, while granularity was specifically increased in LNSC from RA patients $(n=9)$. Initial stainings indicate higher SA $\beta$ gal activity and more DNA damage in LNSC from RA-risk $(n=3)$ and RA patients $(n=4)$ compared with healthy controls $(n=3)$. Expression levels of senescence-associated genes such as p21 and p53 were significantly higher in LNSC from RA patients compared with healthy controls ( $\mathrm{n}=6$ per group).

Conclusion: These preliminary findings suggest senescence already in early RA and provide a rationale for investigating the consequence of senescent LNSC on immune cell responses

Disclosure of Interests: None declared

DOI: 10.1136/annrheumdis-2021-eular.2501

\section{POS0392 PRESENCE OF FOUR SERUM AUTOANTIBODIES ASSOCIATES WITH THE ACPA STATUS IN EARLY RHEUMATOID ARTHRITIS}

L. Lourido ${ }^{1}$, C. Ruiz-Romero ${ }^{1,2}$, L. Collado ${ }^{1}$, M. Hansson ${ }^{3}$, L. Klareskog ${ }^{3}$, R. Sjöberg ${ }^{4}$, E. Pin ${ }^{4}$, P. Nilsson ${ }^{4}$, F. J. Blanco ${ }^{1,5} .{ }^{1}$ Unidad de Proteómica. Grupo de Investigación de Reumatología (GIR)., Instituto de Investigación Biomédica de A Coruña (INIBIC), Complexo Hospitalario Universitario de A Coruña (CHUAC), Sergas. Universidade da Coruña (UDC)., A Coruña, Spain; ${ }^{2}$ Centro de Investigación Biomédica en Red de Bioingeniería, Biomateriales y Nanomedicina, (CIBER-BBN), Madrid, Spain; ${ }^{3}$ Karolinska Institutet, Karolinska University Hospital, Division of Rheumatology, Department of Medicine Solna, Stockholm, Sweden; ${ }^{4} \mathrm{KTH}$ Royal Institute of Technology \& SciLifeLab, Division of Affinity Proteomics, Department of Protein Science, Stockholm, Sweden; ${ }^{5}$ Universidade da Coruña (UDC), Grupo de Investigación de Reumatología y Salud (GIR-S)., Departamento de Fisioterapia, Medicina y Ciencias Biomédicas, Facultad de Fisioterapia, A Coruña, Spain

Background: The presence of anti-citrullinated protein antibodies (ACPAs) is a hallmark of rheumatoid arthritis (RA) that precede the development of the disease by years and is used for its clinical diagnosis. However, there are RA subjects that test negative for ACPA and thus the early diagnosis on these patients may be delayed. Furthermore, the presence or absence of ACPA in RA supports the hypothesis that on these two subsets of patients underlie different pathogenesis and clinical outcomes.

Objectives: In this work, we searched for serum autoantibodies useful to assist the early diagnosis of ACPA-seronegative RA and its management.

Methods: We profiled the serum autoantibody repertoire of 80 ACPA-seronegative and 80 ACPA-seropositive RA subjects from the Swedish population-based Epidemiological Investigation of RA (EIRA) cohort. A suspension bead array platform built on protein fragments within Human Protein Atlas and selected from an initial untargeted screening using arrays containing 2660 total antigens was employed to identify $\lg G$ and IgA serum autoantibodies. A validation phase on antigen suspension bead arrays was carried out on another set of samples from EIRA containing 386 ACPA-seropositive, 358 ACPA-seronegative and 372 randomly selected control subjects of the same age and sex. A sample-specific threshold based on 20 times the median absolute deviation plus the median of al signals was selected to determine the reactivity of samples. The Wilcoxon rank sum test and Fisher's test were applied for the comparison of autoantibody levels and reactivity frequencies between the groups.

Results: Our data revealed four antigens associated with the ACPA status (Table 1). Testis-specific Y-encoded-like protein 4 (TSPYL4) showed significantly higher IgG reactivity frequency in ACPA-seronegative subjects compared to ACPA-seropositive ( $8 \%$ vs. $3 \% ; P<0.05$ ). Significant differences at IgG autoantibody levels $(P<0.05)$ were also observed between ACPA-seronegative subjects and controls for this specific antigen. Significantly higher 